\title{
Computation of Coprime Factorizations of Rational Matrices
}

\author{
A. Varga \\ DLR - Oberpfaffenhofen \\ Institute for Robotics and System Dynamics \\ P.O.B. 1116, D-82230 Wessling, F.R.G. \\ E-mail: Andreas.Varga@dlr.de
}

\begin{abstract}
We propose a numerically reliable state space algorithm for computing coprime factorizations of rational matrices with factors having poles in a given stability domain. The new algorithm is based on a recursive generalized Schur technique for poles dislocation by means of proportional-derivative state feedback. The proposed algorithm is generally applicable regardless the underlying descriptor state space representation is minimal or not, or is stabilizable/detectable or not.
\end{abstract}

\section{Introduction}

Let $G(\lambda)$ be a given $p \times m$ rational matrix. In this paper we address the problem to compute fractional reprezentations of $G$ in the form $G=N M^{-1}$ with $N$ and $M$ rational matrices which are stable and coprime. Such a factorization is called a right coprime factorization (RCF) of $G$. Occasionally additional requirements on the factors may be imposed. Analogously, a fractional representation of $G$ in the form $G=M^{-1} N$, with $N$ and $M$ stable and coprime rational matrices, is called a left coprime factorization (LCF) of $G$. The main applications of the right/left coprime factorizations are in solving various factorization problems encountered in the theory of linear systems and networks. Therefore we assimilate $G(\lambda)$ with the transfer-function matrix (TFM) of a linear time-invariant continuous-time or discrete-time descriptor system and $\lambda$ is either $s$ or $z$, the complex variables appearing in the Laplace- or Z-transform, respectively, in accordance with the type of the system.

In this paper we propose a numerically reliable algorithm to compute RCFs with factors having poles in a specified stability domain $\Gamma$. Depending on the given $\Gamma$, different kinds of RCFs can be computed with the proposed algorithm. If $\Gamma$ contains finite complex numbers, then the resulting denominator $M$ can be always determined as a proper TFM (with $M(\infty)$ finite), but $N$ results proper or improper depending on if the original $G$ is proper or not. The proposed method can be employed to compute RCFs with proper least or- der $M$. Such factorization is useful as a preliminary or as a final steps in computing some other more special factorizations. Proper rational right coprime factorizations (PRRCFs) in which both $N$ and $M$ are proper TFMs are potentially useful in performing order reduction of descriptor systems by using the coprime factors reduction approach analogously as in case of standard systems [1]. Polynomial right comprime factorizations (PORCFs) in which both $N$ and $M$ are polynomial matrices result by choosing $\Gamma=\{\infty\}$. The PORCF is important in several system theoretical computations [2], as for instance in obtaining equivalent polynomial representations of linear systems.

The proposed computational algorithm uses the equivalent descriptor system (or generalized state space) representations of rational matrices. It is based on a recursive pole dislocation technique by means of proportional-derivativative state feedback. The dislocation of poles is performed recursively by using a generalized Schur technique representing an extention of the pole assignment algorithm for descriptor systems proposed in [3]. The new algorithm represents a significant functional enhancement of a similar algorithm using only proportional state feedback [4]. With a single algorithm, different RCFs can be computed by appropriately choosing the stability domain $\Gamma$ and the desired properties for the factors. The new procedure is generally applicable regardless the original descriptor state space representation is minimal or not, or is stabilizable/detectable or not. It is well suited for robust software implementation. The same algorithm can be also employed to compute LCFs by applying it to the dual TFM $G^{T}$.

The proposed algorithm belongs to the class of recursive descriptor state space methods. The recursive approach to rational matrix factorization goes back to Belevitch [5] and more recently Vandewalle and Dewilde [6]. The general technique used in this "transfer-function" approach is the dislocation of poles (or zeros) by using pre- or post-multiplications with elementary first or second order rational factors. The major disadvantage of the above approach, as pointed out in [7], is its compu- 
tational complexity. Typically, the involved computations consist of the computation of poles (or zeros), rational matrix divisions and updating. These disadvantages suggested an alternative "state-space" approach, as advocated by Van Dooren [7]. The techniques developed in this paper can be seen as extensions and refinements of the recursive pole dislocation approach described in [7].

The paper is organized as follows. The main definitions and notations used throughout the paper are presented in a preliminary section. General descriptor system techniques to compute and update fractional representations by using proportional-derivative state feedback are discussed in section 3 . In section 4 we present a recursive procedure for computing RCFs for arbitrary stability domains. This procedure can be also employed to compute proper as well as polynomial RCFs. Some conclusions summarize the main aspects of the proposed computational method.

\section{Preliminaries}

Given a region $\Gamma$ of the complex plane, we call $G \Gamma$ stable if all its poles lie in $\Gamma$. The usual interpretation of $\Gamma$ related to the standard stability concept is the open left complex half plane for a continuous-time system or the open unit disk in the origin for a discrete-time system. We assume throughout the paper that $\Gamma$ is a symmetric region with respect to the real axis, that is, if $\lambda \in \Gamma$ then $\bar{\lambda} \in \Gamma$.

It is well known that any rational matrix $G$ (even improper or polynomial) has a descriptor realization $G=$ $(E, A, B, C, D)$, where $\lambda E-A$ is regular $(\operatorname{det}(\lambda E-A) \not \equiv$ $0)$ and

$$
G(\lambda)=C(\lambda E-A)^{-1} B+D .
$$

For the above descriptor realization we shall also use the alternative notation

$$
G=\left[\begin{array}{c|c}
A-\lambda E & B \\
\hline C & D
\end{array}\right] .
$$

The descriptor representation of $G$ is minimal if the order $n$ of the square matrices $E$ and $A$ has the least value among all possible realizations of $G$. Well known criteria for minimality of a descriptor realization $(E, A, B, C, D)$ are [8]: (i) $\operatorname{rank}[A-\lambda E B]=n$ $\forall \lambda \in \mathbb{C}, \lambda$ finite, and $\operatorname{rank}\left[\begin{array}{ll}E & B\end{array}\right]=n$ (controllability); (ii) $\operatorname{rank}\left[A^{T}-\lambda E^{T} C^{T}\right]^{T}=n \forall \lambda \in \mathbb{C}, \lambda$ finite, and $\operatorname{rank}\left[\begin{array}{ll}E^{T} & C^{T}\end{array}\right]^{T}=n$ (observability); (iii) $A \operatorname{ker}(E) \subseteq \operatorname{range}(E)$ (no nondynamic modes). The descriptor realization $G=(E, A, B, C, D)$ is $\Gamma$-stabilizable if $\operatorname{rank}[A-\lambda E B]=n \forall \lambda \in \Gamma, \lambda$ finite and is $\Gamma$ detectable if $\operatorname{rank}\left[A^{T}-\lambda E^{T} C^{T}\right]^{T}=n \forall \lambda \in \Gamma, \lambda$ finite. The McMillan degree $\delta_{M}(G(\lambda))$ of a rational TFM $G(\lambda)$ is the number of finite and infinite poles of $G(\lambda)$. If the descriptor realization $G=(E, A, B, C, D)$ is minimal, then the finite and infinite poles of $G(\lambda)$ are the finite and infinite zeros of the pencil $A-\lambda E$, respectively. Note that for a minimal descriptor realization of order $n$ we have $n=\delta_{M}(G(\lambda))+\kappa$, where $\kappa$ is the number of infinite elementary divisors of $A-\lambda E$.

A fractional representation of $G$ in the form $G=$ $N M^{-1}$ with $N$ and $M \Gamma$-stable rational matrices, is called a right coprime factorization (RCF) if there exist $\Gamma$-stable rational matrices $U$ and $V$ such that $U N+V M=I$. Of particular interest are the RCFs with least order denominator. We say that the denominator $M$ of a RCF $G=N M^{-1}$ has least order if the McMillan degree of $M$ equals the number of $\Gamma$-unstable poles of $G$.

\section{Fractional Representations: Basic Facts}

Let $G=(E, A, B, C, D)$ be a regular descriptor realization of the rational matrix $G$ and let denote $r=$ $\operatorname{rank}(E)$. The factorization algorithm proposed in this paper relies on simple facts concerning fractional representations. The first result shows how to compute RCFs by using proportional-derivative state feedback.

Fact 1. Any rational matrix $G$ with a $\Gamma$-stabilizable state-space realization $(E, A, B, C, D)$ has a $R C F$ given by the following choice of the factors [9]

$$
\left[\begin{array}{l}
N \\
M
\end{array}\right]=\left[\begin{array}{cc|c}
I & \lambda K & 0 \\
0 & A+B F-\lambda(E-B K) & B W \\
\hline D & C+D F & D W \\
I & F & W
\end{array}\right],
$$

where the state feedback matrices $F$ and $K$ are chosen such that all finite eigenvalues of the pair $(E-B K, A+$ $B F)$ lie in $\Gamma$, the pencil $A+B F-\lambda(E-B K)$ is regular, and $W$ is an arbitrary invertible matrix.

To simplify notations, we shall use in what follows instead (2) the algebraically equivalent more compact representation of factors

$$
\left[\begin{array}{c}
N \\
M
\end{array}\right]=\left[\begin{array}{c|c}
A+B F-\lambda(E-B K) & B W \\
\hline C+D F+\lambda D K & D W \\
F+\lambda K & W
\end{array}\right] .
$$

The matrices $F, K$ and $W$ can be viewed as free parameters which determine a particular factorization. Factorizations with special properties, as for instance with proper or polynomial factors, can be determined by suitably choosing the matrix pair $(F, K)$. Although for the RCFs considered in this paper the input scaling matrix $W$ can be always chosen as $W=I$, we consider in what follows the more general case of arbitrary invertible $W$, because of its potential interest for new algorithms.

The algorithm proposed in this paper uses implicitly 
the more general expressions for the factors

$$
\left[\begin{array}{c}
N \\
M
\end{array}\right]=\left[\begin{array}{c|c}
U(A+B F-\lambda(E-B K)) V & U B W \\
\hline(C+D(F+\lambda K)) V & D W \\
(F+\lambda K) V & W
\end{array}\right],
$$

where $U$ and $V$ are orthogonal transformation matrices (usually not explicitly accumulated). Although general, nonsingular matrices $U$ and $V$ could be also considered as additional free parameters of RCFs, their role in the proposed algorithms is only to allow to obtain the resulting matrices in particular condensed forms or to preserve convenient condensed forms of matrices which ensure an efficient implementation of the algorithms.

Fact 2. If $G=N_{1} M_{1}^{-1}$ and $N_{1}=N_{2} M_{2}^{-1}$, then $G$ has the fractional representation $G=N M^{-1}$, where $N=N_{2}$ and $M=M_{1} M_{2}$.

This simple fact allows us to obtain explicit formulae to update partial factorizations by using simple state space formulae. Let $N_{1}$ and $M_{1}$ be the factors computed as

$$
\left[\begin{array}{l}
N_{1} \\
M_{1}
\end{array}\right]=\left[\begin{array}{c|c}
A+B F_{1}-\lambda\left(E-B K_{1}\right) & B W_{1} \\
\hline C+D F_{1}+\lambda D K_{1} & D W_{1} \\
F_{1}+\lambda K_{1} & W_{1}
\end{array}\right]
$$

and let $N_{2}$ and $M_{2}$ be the factors of $N_{1}$ computed as

$$
\left[\begin{array}{c}
N_{2} \\
M_{2}
\end{array}\right]=\left[\begin{array}{c|c}
A+B F-\lambda(E-B K) & B W \\
\hline C+D F+\lambda D K & D W \\
F_{2}+\lambda K_{2} & W_{2}
\end{array}\right],
$$

where

$$
\begin{aligned}
& F=F_{1}+W_{1} F_{2} \\
& K=K_{1}+W_{1} K_{2} \\
& W=W_{1} W_{2} .
\end{aligned}
$$

It easy to verify that the product $M_{1} M_{2}$ is given by $M_{1} M_{2}=(E-B K, A+B F, B W, F+\lambda K, W)$ and thus equations (7) serve as explicit updating formulae of fractional representations. These formulae can be extended easily to include arbitrary coordinate transformation matrices.

Updating formulae can be also used at the level of the system matrices which defines a coprime factorization. If we denote $\widetilde{E}=E-B K_{1}, \widetilde{A}=A+B F_{1}, \widetilde{B}=B W_{1}$, $\widetilde{C}=C+D F_{1}, \widetilde{B}=B W_{1}$ and $\widetilde{D}=D W_{1}$, then the following formulae can be used simultaneously to update $\widetilde{E}, \widetilde{A}, \widetilde{B}, \widetilde{C}$ and $\widetilde{D}$ :

$$
\begin{array}{ll}
\widetilde{E} & \leftarrow \widetilde{E}-\widetilde{B} K_{2}, \\
\widetilde{A} \leftarrow \widetilde{A}+\widetilde{B} F_{2}, & \widetilde{B} \leftarrow \widetilde{B} W_{2}, \\
\widetilde{C} \leftarrow \widetilde{C}+\widetilde{D} F_{2}, \quad \widetilde{D} \leftarrow \widetilde{D} W_{2} .
\end{array}
$$

The factorization algorithm presented in the paper relies on the use of such updating formulae. For $W_{1}=I$, $W_{2}=I$, the updating formulae (7) reduce to simple matrix additions

$$
\begin{aligned}
& F=F_{1}+F_{2} \\
& K=K_{1}+K_{2},
\end{aligned}
$$

which are used in the proposed algorithm.

\section{RCF with $\Gamma$-stable factors}

Let $G$ be a given rational matrix and let $\Gamma$ be a given stability region of the complex plane. In this section we propose an algorithm to compute a RCF $G=N M^{-1}$ with the factors $N$ and $M$ having poles only in $\Gamma$. This algorithm is based on numerically reliable computations and, additionally, it can even handle the case when the original descriptor system representation is not $\Gamma$ stabilizable. The basis for our algorithm is a pole assignment procedure described in [3], which is extended to handle proportional+derivative state feedback. This algorithm has the ability to keep unaltered the eigenvalues of the pair $(E, A)$ which already lie in $\Gamma$ and to move only those outside of $\Gamma$ to locations within $\Gamma$ by choosing appropriate proportional and derivative feedback matrices $F$ and $K$, respectively. An additional useful feature of this algorithm is that simultaneously with the stabilizing pair $(F, K)$, it determines the generalized Schur form of the pair $(E-B K, A+B F)$. This makes possible to extract easily a minimal descriptor realization for the denominator factor $M$.

The main steps of the generalized Schur algorithm are shortly explained below. Assume that the pair $(E, A)$ is already in a generalized real Schur form (GRSF), and the matrices $E, A$ and $B$ are partitioned conformally as

$$
E=\left[\begin{array}{cc}
E_{11} & E_{12} \\
0 & E_{22}
\end{array}\right], A=\left[\begin{array}{cc}
A_{11} & A_{12} \\
0 & A_{22}
\end{array}\right], B=\left[\begin{array}{l}
B_{1} \\
B_{2}
\end{array}\right],
$$

where the pair $\left(E_{22}, A_{22}\right)$ has only generalized eigenvalues outside $\Gamma$. By choosing the feedback matrices analogously partitioned as

$$
F=\left[\begin{array}{ll}
0 & F_{2}
\end{array}\right], \quad K=\left[\begin{array}{ll}
0 & K_{2}
\end{array}\right]
$$

we see that the pair $(E-B K, A+B F)$ has the form

$$
\left(\left[\begin{array}{cc}
E_{11} & E_{12}-B_{1} K_{2} \\
0 & E_{22}-B_{2} K_{2}
\end{array}\right],\left[\begin{array}{cc}
A_{11} & A_{12}+B_{1} F_{2} \\
0 & A_{22}+B_{2} F_{2}
\end{array}\right]\right)
$$

and thus, the feedback perturbed only the generalized eigenvalues of the pair $\left(E_{22}, A_{22}\right)$, leaving the rest of generalized eigenvalues of the pair $(E, A)$ unmodified. In particular, if $E_{22}$ and $A_{22}$ are the last diagonal blocks in the GRSF (of order one or two), then the pair $(E-B K, A+B F)$ is further in a GRSF. Provided $B_{2} \neq 0$, the generalized eigenvalues of the pair $\left(E_{22}-B_{2} K_{2}, A_{22}+B_{2} F_{2}\right)$ can be arbitrarily modified by suitably choosing $F_{2}$ and $K_{2}$.

The stabilization within $\Gamma$ of a given system can be performed by iteratively modifying the generalized eigenvalues of the pair $(E, A)$ as in the following conceptual algorithm:

1. Reduce the system matrices by using orthogonal similarity transformations such that the pair $(E, A)$ is 
in an ordered GRSF (10) with the pair $\left(E_{22}, A_{22}\right)$ having only generalized eigenvalues in $\Gamma$ and the pair $\left(E_{11}, A_{11}\right)$ having only generalized eigenvalues in $\mathbb{C} \backslash \Gamma$.

2. Determine the feedback matrices $F$ and $K$ of the form (11) which moves the generalized eigenvalues of the last diagonal blocks of $(E, A)$ to locations within $\Gamma$.

3. Update $E$ as $E-B K$ and $A$ as $A+B F$; by using orthogonal similarity transformations, bring another pair of diagonal blocks of $(E, A)$ with eigenvalues outside $\Gamma$ in the last diagonal position and resume the previous step.

Note that if $\Gamma$ includes at least one finite element then proportional state feedback alone is sufficient to move the finite eigenvalues of the pair $(E, A)$ to arbitrary finite locations within $\Gamma$. If $\Gamma=\{\infty\}$ and $A$ is nonsingular then derivative feedback alone is sufficient to move all finite eigenvalues to infinity. However in this latter case, in general, both proportional and derivative feedback are necessary to ensure the regularity of the resulting factors.

To ensure complete generality, a deflation mechanism can be easily included into the factorization algorithm to remove automatically the $\Gamma$-unstabilizable part of the system. Such deflation is possible by observing that if the generalized eigenvalues corresponding to the last diagonal blocks $E_{22}$ and $A_{22}$ are not controllable, then the corresponding $B_{2}$ should be zero. If we partition $C$ analogously with the matrices in (10) as $C=\left[C_{1} C_{2}\right]$, then we can replace the original descriptor system $(E, A, B, C, D)$ with an input-output equivalent realization of lower order $\left(E_{11}, A_{11}, B_{1}, C_{1}, D\right)$ by simply deleting the rows and columns in matrices $E, A$, $B$ and $C$ which correspond to the unstabilizable part. In this case the resulting coprime factorization has order less than $n$.

The following implementable state space algorithm to compute a RCF of a rational TFM $G$ materializes the above ideas.

\section{GRCF-PD Algorithm.}

1. Reduce the pair $(E, A)$ by an orthogonal similarity transformation, to the ordered GRSF [10], [11] $(\widetilde{E}, \widetilde{A})=(Q E Z, Q A Z)$, partitioned as

$$
\widetilde{E}=\left[\begin{array}{cc}
E_{11} & E_{12} \\
0 & E_{22}
\end{array}\right], \widetilde{A}=\left[\begin{array}{cc}
A_{11} & A_{12} \\
0 & A_{22}
\end{array}\right]
$$

where $E_{11}, A_{11} \in \mathbb{R}^{q \times q}, Q$ and $Z$ are orthogonal matrices, $\Lambda\left(A_{11}, E_{11}\right) \subset \Gamma$ and $\Lambda\left(A_{22}, E_{22}\right) \subset \mathbb{C} \backslash \Gamma$. Compute $\widetilde{B}=Q B, \widetilde{C}=C Z$, and set $\widetilde{F}=0$ and $\widetilde{K}=0$.

2. If $q=n$, go to step 7 .

3. Let $(\delta, \alpha)$ be the pair of last elementary diagonal blocks of order $k(k=1$ or 2$)$ of the pair $(\widetilde{E}, \widetilde{A})$ in GRSF and let $\beta$ be the matrix formed from the last $k$ rows of $\widetilde{B}$. If $\|\beta\| \leq \epsilon$ (a given tolerance), then $n \leftarrow n-k$ and go to step 2 .

4. Compute $\varphi$ and $\kappa$ such that $\Lambda(\delta-\beta \kappa, \alpha+\beta \varphi) \subset \Gamma$.

5. Compute $\widetilde{E} \leftarrow \widetilde{E}-\widetilde{B}[0 \kappa], \widetilde{A} \leftarrow \widetilde{A}+\widetilde{B}[0 \varphi], \widetilde{F} \leftarrow$ $\widetilde{F}+[0 \varphi], \widetilde{K} \leftarrow \widetilde{K}+[0 \kappa]$.

6. Compute the orthogonal similarity transformation matrices $\widetilde{Q}$ and $\widetilde{Z}$ to reorder the diagonal blocks of the pair $(\widetilde{E}, \widetilde{A})$ in GRSF, such that the last block of $(\widetilde{E}, \widetilde{A})$ is moved by successive interchanging of diagonal blocks to row position $q+1$. Compute $\widetilde{E} \leftarrow \widetilde{Q} \widetilde{E} \widetilde{Z}$, $\widetilde{A} \leftarrow \widetilde{Q} \widetilde{A} \widetilde{Z}, \widetilde{B} \leftarrow \widetilde{Q} \widetilde{B}, \widetilde{C} \leftarrow \widetilde{C} \widetilde{Z}, \widetilde{F} \leftarrow \widetilde{F} \widetilde{Z}, \widetilde{K} \leftarrow \widetilde{K} \widetilde{Z}$. Put $q \leftarrow q+k$ and go to step 2 .

7. Set

$$
\left[\begin{array}{l}
N \\
M
\end{array}\right]=\left[\begin{array}{c|c}
\widetilde{A}-\lambda \widetilde{E} & \widetilde{B} \\
\hline \widetilde{C}+\underset{\widetilde{F}+\lambda D \widetilde{K}}{D \widetilde{F}+\lambda \widetilde{K}} & D \\
I
\end{array}\right]
$$

Algorithm GRCF-PD can be easily constrained to use only proportional or only derivative state feedback. We shall refer to the corresponding algorithmic variants as Algorithm GRCF-P and Algorithm GRCF-D, respectively.

This algorithm can be viewed as a recursive updating procedure of an initial fractional representation $G=N_{0} M_{0}^{-1}$ with $N_{0}=G$ and $M_{0}=I$, by using the updating formulae (8) and (9) combined with orthogonal coordinate transformations performed on the matrices of partial factorizations. The matrix pair $(\widetilde{E}, \widetilde{A})$ in the initial factorization of $G$ is in a GRSF (computed at step 1) and this form is preserved at subsequent steps. The resulting final pair $(\widetilde{E}, \widetilde{A})$ is in a GRSF too and, if the original system is $\Gamma$-stabilizable, then $\widetilde{E}$ and $\widetilde{A}$ contain the matrices $U(E-B K) V$ and $U(A+B F) V$, respectively, where $U$ and $V$ are the accumulated orthogonal transformations performed at steps 1 and 6 of the algorithm, and $F$ and $K$ are the stabilizing feedback matrices $\widetilde{F} V^{T}$ and $\widetilde{K} V^{T}$, respectively. If the original system is not $\Gamma$-stabilizable, then the unstabilizable blocks are detected at step 3 and the corresponding unstabilizable parts are deflated by simply decreasing the order of system with $k$. If unstabilizable blocks are detected by the algorithm, then the resulting state vector dimension of the numerator $N$ is less than $n$.

The particular structures of matrices resulting from the above algorithm allow to determine easily a minimal realization of $M$. The resulting $\widetilde{F}$ and $\widetilde{K}$ have always the forms

$$
\widetilde{F}=\left[0 \widetilde{F}_{2}\right], \quad \widetilde{K}=\left[\begin{array}{ll}
0 & \widetilde{K}_{2}
\end{array}\right]
$$

where the number of columns of $\widetilde{F}_{2}$ and $\widetilde{K}_{2}$ is equal to the number of $\Gamma$-unstable controllable generalized 
eigenvalues of the pair $(E, A)$. If we partition accordingly the resulting $\widetilde{E}, \widetilde{A}$ and $\widetilde{B}$

$$
\widetilde{E}=\left[\begin{array}{cc}
\widetilde{E}_{11} & \widetilde{E}_{12} \\
0 & \widetilde{E}_{22}
\end{array}\right], \widetilde{A}=\left[\begin{array}{cc}
\widetilde{A}_{11} & \widetilde{A}_{12} \\
0 & \widetilde{A}_{22}
\end{array}\right], \widetilde{B}=\left[\begin{array}{c}
\widetilde{B}_{1} \\
\widetilde{B}_{2}
\end{array}\right],
$$

then $\left(\widetilde{E}_{22}, \widetilde{A}_{22}, \widetilde{B}_{2}, \widetilde{F}_{2}+\lambda \widetilde{K}_{2}, I\right)$ is a minimal realization of $M$. If $\widetilde{E}_{22}$ is invertible, then $M$ is proper, while if $\widetilde{E}_{22}$ is nilpotent, then $M$ is polynomial.

Algorithm GRCF-PD is quite flexible in coping with different computational tasks. If all assigned eigenvalues from $\Gamma$ are finite, then $\widetilde{E}_{22}$ is invertible and the TFM $M$ results proper. However, in general, the factor $N$ is not proper if the original $G$ is not proper. To enforce the properness of $N$, we can first use Algorithm GRCF-D to compute a fractional representation $G=N_{1} M_{1}^{-1}$ with $N_{1}$ and $M_{1}$ proper, but possibly $\Gamma$ unstable. Then, we can apply Algorithm GRCF-P to the TFM $\left[\begin{array}{l}N_{1} \\ M_{1}\end{array}\right]$ to obtain the $\operatorname{RCF}\left[\begin{array}{l}N_{1} \\ M_{1}\end{array}\right]=\left[\begin{array}{l}N \\ M\end{array}\right] M_{2}$ from which the PRRCF of $G$ results as $G=N M^{-1}$. The first step of this approach requires the reliable numerical separation of the finite and infinite structures of the regular pencil $A-\lambda E$ at step 1 of Algorithm GRCFD. For this purpose, instead eigenvalues reordering, a more robust numerical approach is recommended (see for example [12]). The resulting McMillan degree of $M$ is usually larger then the minimal one. An alternative approach to compute a PRRCF by using only proportional feedback is presented in [4].

Another interesting application is the computation of PORCFs. In this case, by setting $\Gamma=\{\infty\}$ both factors $N$ and $M$ are polynomial matrices because $\widetilde{E}$ results nilpotent. The role of the derivative feedback is to move all eigenvalues of $E-B K$ to the origin, while the proportional feedback is used only occasionally to ensure the regularity requirement in the case when $G$ has poles in the origin. The McMillan degree of the resulting $M$ is the least one, provided the original descriptor realization of $G$ has all finite eigenvalues observable (uncontrollable finite eigenvalues are automatically removed). In this case the least possible McMillan degree is precisely the number of finite poles of $G$.

Algorithm GRCF-P can be generally used if $\Gamma$ contains finite elements. In this case, if in the descriptor representation of $G$ the $\Gamma$-unstable controllable eigenvalues of the pair $(E, A)$ are observable, then the order of the minimal realization of $M$ is equal to the least possible McMillan degree for $M$, which is precisely the number of $\Gamma$-unstable poles of $G$. However, although the resulting descriptor representation of $M$ is always minimal, the McMillan degree of $M$ can be higher than the least possible McMillan degree if some $\Gamma$-unstable eigenvalues of $(E, A)$ are controllable but unobservable.

The GRCF-PD Algorithm is based on a generalization of a pole assignment algorithm for standard systems
[13]. A tentative roundoff error analysis of that algorithm [14] indicates that if each partial feedback matrix $\varphi$ (as that computed at step 4 for instance) satisfies the condition $\|\varphi\| \leq\|A\| /\|B\|$, then the pole assignment algorithm is numerically backward stable. It is likely that similar conditions on the partial feedbacks $\varphi$ and $\kappa$ can guarantee the numerical reliability of the proposed algorithm too. This aspect needs certainly further investigations. We note however that unfortunately this condition can not be always fulfilled if large gains are necessary to stabilize the system. This can arise either if the unstable poles are too "far" from the $\Gamma$-region or if these poles are weekly controllable.

\section{Conclusions}

We proposed an efficient, numerically reliable descriptor state space algorithm for computing RCFs of rational matrices. The proposed algorithm is based on a recursive generalized Schur technique for poles dislocation by using proportional+derivative state feedback. The use of the derivative feedback allows to compute for instance RCFs with proper or polynomial factors. The new algorithm is completely general and has no restrictions on the properties of the underlying descriptor representations. It is well suited for a robust and modular software implementation.

\section{References}

[1] Y. Liu and B. D. O. Anderson. Controller reduction via stable factorization and balancing. Int. J. Control, 44:507-531, 1986.

[2] H. Kwakernaak. State space algorithms for polynomial matrix computations. In Proc. EURACO Workshop, Florence, Italy, pp. 133-170, 1995.

[3] A. Varga. On stabilization of descriptor systems. Systems \& Control Letters, 24:133-138, 1995.

[4] A. Varga. Generalized Schur methods to compute coprime factorizations of rational matrices. In Proc. 1st Asian Control Conference, Tokyo, Japan, vol. 3, pp. 8992, 1994.

[5] V. Belevitch. Classical Network Theory. Holden Day, San Francisco, 1968.

[6] J. Vandewalle and P. Dewilde. On the irreducible cascade synthesis of a system with real rational transfer matrix. IEEE Trans. Circuits and Systems, 24:481-494, 1977.

[7] P. Van Dooren. Rational and polynomial matrix factorizations via recursive pole-zero cancellation. Lin. Alg. \& Appl., 137/138:663-697, 1990.

[8] G. Verghese, B. Lévy, and T. Kailath. A generalized state-space for singular systems. IEEE Trans. Autom. Control, AC-26:811-831, 1981.

[9] F.R. Chang, C.H. Fang, and C.H. Wang. Doubly coprime matrix-fraction representations using pro- 
portional and derivative feedback concepts in generalized state-space systems. IEEE Trans. Autom. Control, 36:1193-1195, 1991.

[10] C. B. Moler and G. W. Stewart. An algorithm for generalized matrix eigenvalue problem. SIAM J. Numer. Anal., 10:241-256, 1973.

[11] P. Van Dooren. A generalized eigenvalue approach for solving Riccati equations. SIAM J. Sci. Stat. Comput., 2:121-135, 1981.

[12] A. Varga. Computation of transfer function matrices of generalized state-space models. Int. J. Control, 50:2543-2561, 1989.

[13] A. Varga. A Schur method for pole assignment. IEEE Trans. Autom. Control, AC-26:517-519, 1981.

[14] A. Varga. The numerical stability of an algorithm for pole assignment. In G. Leininger (Ed.), Proc. 2-nd IFAC CADCS Symp., West Lafayette, IN, pp. 117-122. Pergamon Press, Oxford, 1982. 